\title{
Relation between initial treatment strategy in stable coronary artery disease and 1-year costs in Ontario: a population-based cohort study
}

\author{
Jaskaran S. Kang MD, Maria C. Bennell MPH, Feng Qiu MSc, Merril L. Knudtson MD, \\ Peter C. Austin PhD, Dennis T. Ko MD MSc, Harindra C. Wijeysundera MD PhD
}

Abstract

Background: Cardiovascular disease is costly, and annual expenditures are projected to increase. Our objective was to examine the variation in patient-level costs and identify drivers of cost in patients with stable coronary artery disease.

\begin{abstract}
Methods: In this retrospective cohort study using administrative databases in Ontario, Canada, we identified all patients with stable coronary artery disease after index angiography between Oct. 1, 2008, and Sept. 30, 2011. We excluded patients with a myocardial infarction within 90 days before the index, with normal coronaries, or with mild coronary disease. We categorized hospitals into low, medium or high revascularization ratio centres. The primary outcome was cumulative 1-year health care costs. A hierarchical generalized linear model identified patient, physician and hospital characteristics associated with patient costs, with 2 main covariates of interest: treatment allocation (medical v. percutaneous coronary intervention v. coronary artery bypass grafting) and hospital revascularization ratio.
\end{abstract}

Results: A total of 183630 angiography procedures were performed in Ontario during the study period. The final cohort included 39126 patients with stable coronary artery disease, of which 15138 received medical treatment and 23988 received revascularization. The mean 1-year cost was $\$ 24026$ (interquartile range $\$ 8235-\$ 30511$ ). The mean costs for medical management and revascularization were $\$ 18069$ and $\$ 27786$, respectively. The strongest predictor of costs was revascularization (percutaneous coronary intervention: cost ratio $1.27,95 \% \mathrm{Cl}$ [confidence interval] 1.24-1.31; coronary artery bypass grafting: cost ratio $2.62,95 \% \mathrm{Cl}$ 2.53-2.71). Hospital revascularization ratio did not significantly affect costs. There was no significant interaction between treatment and revascularization ratio.

Interpretation: Most health care costs were due to acute care hospital admissions, and costs were higher for patients undergoing revascularization than medical therapy. This study suggests that treatment decision has a substantial impact on health care resources.

oronary artery disease is a leading cause of morbidity and mortality, ${ }^{1}$ and as such represents a major economic burden on health care expenditures. ${ }^{2}$ The cost of cardiovascular disease and stroke is $\$ 7.6$ billion in Canada for direct health care costs annually and $\$ 14.6$ billion for indirect costs of disability or death, with expenditures projected to increase. ${ }^{3}$

Treatment for stable coronary artery disease includes medical therapy alone or in combination with revascularization by either percutaneous coronary intervention or coronary artery bypass grafting. There is ongoing controversy as to the best initial treatment strategy despite numerous landmark trials that suggest the 2 treatment choices are equivalent with regard to death, major adverse cardiovascular events and symptom relief. ${ }^{46}$ This debate has resulted in significant variation in clinical practice. ${ }^{7}$ Understanding the potential impact of such practice variation on resource use is important. This is especially relevant given the current era of substantial budgetary constraints, where there is high priority for the efficient use of scarce health care resources. Indeed, recent guidelines from professional cardiovascular societies reinforce the importance of incorporating value and resource implications into health care decisions. ${ }^{8}$

Although there are studies examining the predictors of initial treatment strategy in patients with stable coronary artery disease, there is a paucity of literature on the impact of the different treatment strategies on health care costs. Accordingly, we sought to address this gap in knowledge by studying the cumulative 1-year health care costs in patients diagnosed with stable

Competing interests: Harindra Wijeysundera reports grants from Edwards Lifesciences. No other competing interests were declared.

This article has been peer reviewed.

Correspondence to: Harindra Wijeysundera, harindra.wijeysundera@ sunnybrook.ca

CMAJ Open 2016. DOI:10.9778/cmajo.20150138 
coronary artery disease after a coronary angiogram, using a population-based clinical registry of patients in Ontario, Canada. Ontario is Canada's largest province with about 14 million residents, all of whom receive universal health coverage provided by a single third-party payer, the Ontario Ministry of Health and Long-term Care. Our objectives were to determine the degree of variation in health care costs and understand the predictors of cumulative health care costs. We were interested in the relation between initial treatment strategy (medical management $\mathrm{v}$. revascularization) as well as the impact of the hospital revascularization ratio on subsequent health care use.

\section{Methods}

This observational cohort study was approved by the institutional review board at Sunnybrook Health Sciences Centre in Toronto, Ontario. Under Ontario's Personal Health Information Protection Act, the need for patient consent was waived.

\section{Setting}

Data were obtained from the Cardiac Care Network of Ontario, which is a network of 19 hospitals that provide adult cardiac services, and includes a clinical registry of patients who undergo cardiac angiography, percutaneous coronary intervention or coronary artery bypass grafting. ${ }^{9,10}$ Its accuracy has been validated by retrospective chart review. ${ }^{11,12} \mathrm{We}$ linked data from the network to population-level administrative databases using unique, encoded identifiers and analyzed the data at the Institute for Clinical Evaluative Sciences. ${ }^{13-16}$ See Appendix 1 (available at www.cmajopen.ca/content/4/3/ E409/suppl/DC1) for details of the administrative databases.

\section{Patients}

The cohort consisted of patients who underwent angiography between Oct. 1, 2008, and Sept. 30, 2011, for the indication of stable coronary artery disease, and who had obstructive coronary stenosis. During this period, there were only 18 member hospitals in the Cardiac Care Network of Ontario. For patients who underwent angiography multiple times during the study period, we considered only the initial angiogram. We excluded patients who had a myocardial infarction within 90 days before the first angiogram, those with normal coronary arteries, those with mild coronary artery disease and patients with missing angiography data.

\section{Exposure}

We allocated patients who received revascularization within 90 days of their index angiogram to the revascularization strategy group (and into percutaneous coronary intervention or coronary artery bypass grafting subgroups), as is consistent with the literature and known procedural wait times, ${ }^{10,11}$ and we allocated all remaining patients to the medical therapy strategy group.

\section{Ratio of revascularization to medical therapy}

We calculated the ratio of revascularization to medical therapy (referred to herein as the revascularization ratio), defined as the number of patients who received revascularization by the num- ber of patients who underwent medical therapy. We then allocated the hospitals equally into 1 of 3 categories: high, medium, or low, based on equal tertiles of revascularization ratio.

\section{Main outcomes}

The primary outcome was the total cumulative cost per patient in the 1 year following the index angiography. Complete cost profiles were available for all patients for 1 year or until death. As such, we did not have to consider censored costs in our analyses. The categories of costs were all-cause physician visits and laboratory tests, acute care and chronic care hospital admissions, emergency department visits, sameday surgeries and coronary artery disease-related medication use for patients aged 65 years and older. ${ }^{15,16}$

We determined costs associated with physician visits and laboratory tests using data from the claims history of the Ontario Health Insurance Program database. ${ }^{14}$ The database also included shadow billings from providers of organizations covered by alternate payment arrangements. We estimated the cost of hospital admission using the resource intensity weights method. ${ }^{16} \mathrm{We}$ multiplied the resource intensity weights associated with the case-mix group for each hospital admission in the Canadian Institute for Health Information Discharge Abstract Database by the average provincial cost per weighted case for all Ontario acute and chronic care hospitals for that year. ${ }^{16}$ This method yields a mean cost per hospital admission for cases assigned to a particular case-mix group category. We used a similar resource intensity weights

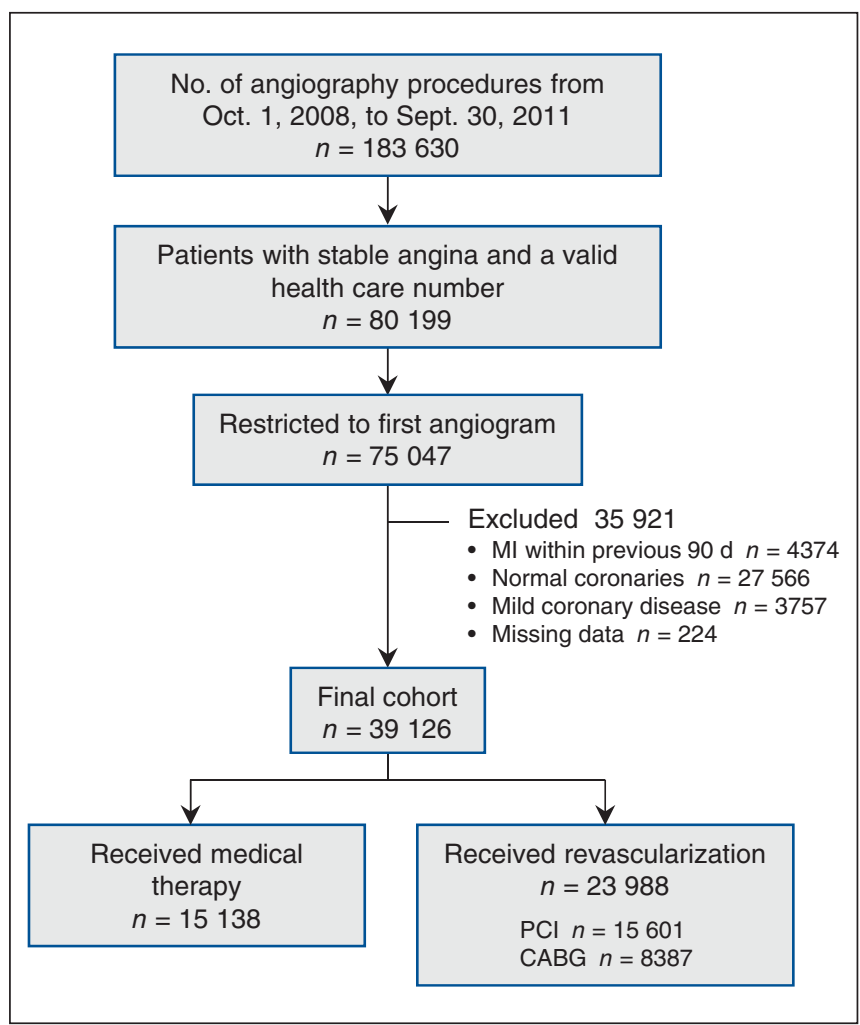

Figure 1: Selection of patients for the study. Note: $C A B G=$ coronary artery bypass grafting, $\mathrm{MI}=$ myocardial infarction, $\mathrm{PCl}$ = percutaneous coronary intervention. 


\begin{tabular}{|c|c|c|c|c|c|c|}
\hline \multirow[b]{2}{*}{ Variable } & \multicolumn{3}{|c|}{$\%$ of patients* } & \multirow{2}{*}{$\begin{array}{c}p \text { value } \\
\text { (medical therapy v. } \\
\text { revascularization) }\end{array}$} & \multicolumn{2}{|c|}{$\%$ of patients ${ }^{*}$} \\
\hline & $\begin{array}{c}\text { Total } \\
n=39126\end{array}$ & $\begin{array}{c}\text { Medical therapy } \\
\quad n=15138\end{array}$ & $\begin{array}{c}\text { Revascularization } \\
n=23988\end{array}$ & & $\begin{array}{c}\mathrm{PCl} \\
n=15601\end{array}$ & $\begin{array}{c}\text { CABG } \\
n=8387\end{array}$ \\
\hline \multicolumn{7}{|l|}{ Patient-level factors } \\
\hline \multicolumn{7}{|l|}{ Demographics } \\
\hline Age, mean \pm SD, yr & $66.0 \pm 10.3$ & $67.0 \pm 10.2$ & $65.3 \pm 10.3$ & $<0.001$ & $65.0 \pm 10.7$ & $66.0 \pm 9.7$ \\
\hline Sex, male & 75.3 & 74.3 & 75.9 & $<0.001$ & 72.9 & 81.3 \\
\hline Rural residence & 14.5 & 14.4 & 14.5 & 0.7 & 14.6 & 14.5 \\
\hline Income quintile & & & & $<0.001$ & & \\
\hline 1 (lowest) & 18.6 & 19.6 & 17.9 & & 18.4 & 17.1 \\
\hline 2 & 20.4 & 20.4 & 20.4 & & 20.4 & 20.6 \\
\hline 3 & 20.2 & 20.3 & 20.2 & & 20.3 & 19.9 \\
\hline 4 & 20.7 & 20.2 & 21.0 & & 20.8 & 21.3 \\
\hline 5 (highest) & 20.1 & 19.4 & 20.5 & & 20.2 & 21.1 \\
\hline \multicolumn{7}{|l|}{ Medical comorbidities } \\
\hline PVD & 9.4 & 11.5 & 8.1 & $<0.001$ & 7.6 & 9.0 \\
\hline Previous MI & 28.1 & 35.8 & 23.3 & $<0.001$ & 24.8 & 20.4 \\
\hline COPD & 6.9 & 8.6 & 5.8 & $<0.001$ & 6.1 & 5.1 \\
\hline $\begin{array}{l}\text { Charlson Comorbidity } \\
\text { Index score, } \\
\text { mean } \pm \text { SD }\end{array}$ & $0.9 \pm 1.3$ & $1.1 \pm 1.5$ & $0.8 \pm 1.2$ & $<0.001$ & $0.7 \pm 1.3$ & $0.8 \pm 1.2$ \\
\hline \multicolumn{7}{|l|}{ Cardiac risk factors } \\
\hline Diabetes & 44.0 & 48.0 & 41.4 & $<0.001$ & 40.5 & 43.1 \\
\hline Hypertension & 86.7 & 89.5 & 84.9 & $<0.001$ & 84.9 & 85.1 \\
\hline Hyperlipidemia & 80.8 & 83.0 & 79.5 & $<0.001$ & 79.9 & 78.7 \\
\hline History of smoking & 31.6 & 33.2 & 30.5 & $<0.001$ & 30.3 & 31.0 \\
\hline \multicolumn{7}{|l|}{ Cardiac status/testing } \\
\hline \multicolumn{7}{|l|}{ Native stenosis $†$} \\
\hline LM & 13.0 & 10.8 & 14.4 & $<0.001$ & 5.5 & 31.0 \\
\hline Proximal LAD & 33.2 & 28.7 & 36.0 & $<0.001$ & 29.7 & 47.6 \\
\hline Mid/distal LAD & 49.6 & 47.5 & 51.0 & $<0.001$ & 46.0 & 60.3 \\
\hline Circumflex & 51.5 & 50.9 & 51.9 & 0.07 & 43.6 & 67.2 \\
\hline $\mathrm{RCA}$ & 60.8 & 60.5 & 60.9 & 0.4 & 54.7 & 72.6 \\
\hline Had previous CABG & & & & $<0.001$ & & \\
\hline Yes & 18.0 & 30.1 & 10.4 & & 15.1 & 1.6 \\
\hline No & 81.9 & 69.9 & 89.5 & & 84.9 & 98.2 \\
\hline LV function & & & & $<0.001$ & & \\
\hline$\leq 34 \%$ & 5.4 & 8.4 & 3.5 & & 3.3 & 4.0 \\
\hline $35 \%-49 \%$ & 12.9 & 16.0 & 11.0 & & 10.6 & 11.7 \\
\hline$\geq 50 \%$ & 48.6 & 46.5 & 50.0 & & 49.7 & 50.5 \\
\hline NA & 33.0 & 29.1 & 35.5 & & 36.4 & 33.8 \\
\hline Exercise ECG risk & & & & $<0.001$ & & \\
\hline High risk & 27.7 & 21.3 & 31.7 & & 28.8 & 37.1 \\
\hline Low risk & 22.4 & 22.9 & 22.2 & & 23.0 & 20.7 \\
\hline Uninterpretable & 4.9 & 4.8 & 4.9 & & 5.3 & 4.2 \\
\hline NA & 45.0 & 51.1 & 41.2 & & 42.9 & 38.0 \\
\hline
\end{tabular}




\section{OPEN}

Research

Table 1 (part 2 of 2): Baseline characteristics of patients with stable angina, by initial treatment strategy

\begin{tabular}{|c|c|c|c|c|c|c|}
\hline \multirow[b]{2}{*}{ Variable } & \multicolumn{3}{|c|}{$\%$ of patients ${ }^{\star}$} & \multirow{2}{*}{$\begin{array}{c}p \text { value } \\
\text { (medical therapy v. } \\
\text { revascularization) }\end{array}$} & \multicolumn{2}{|c|}{$\%$ of patients ${ }^{*}$} \\
\hline & $\begin{array}{c}\text { Total } \\
n=39126\end{array}$ & $\begin{array}{l}\text { Medical therapy } \\
n=15138\end{array}$ & $\begin{array}{l}\text { Revascularization } \\
n=23988\end{array}$ & & $\begin{array}{c}\mathrm{PCl} \\
n=15601\end{array}$ & $\begin{array}{c}\text { CABG } \\
n=8387\end{array}$ \\
\hline \multicolumn{7}{|c|}{ Patient-level factors cont'd } \\
\hline \multicolumn{7}{|c|}{ Cardiac status/testing cont'd } \\
\hline Functional imaging risk & & & & $<0.001$ & & \\
\hline High risk & 32.0 & 31.2 & 32.6 & & 31.1 & 35.3 \\
\hline Low risk & 23.0 & 25.1 & 21.7 & & 23.2 & 19.1 \\
\hline Unknown/NA & 44.9 & 43.7 & 45.7 & & 45.7 & 45.7 \\
\hline CCS class & & & & $<0.001$ & & \\
\hline 0 & 16.5 & 22.0 & 13.0 & & 11.3 & 16.2 \\
\hline 1 & 14.0 & 15.9 & 12.8 & & 12.3 & 13.8 \\
\hline 2 & 38.3 & 35.5 & 40.0 & & 40.8 & 38.5 \\
\hline 3 & 28.5 & 24.2 & 31.1 & & 32.3 & 29.0 \\
\hline 4 & 2.8 & 2.4 & 3.0 & & 3.3 & 2.5 \\
\hline \multicolumn{7}{|l|}{ Physician-level factors } \\
\hline Referral physician & & & & 0.01 & & \\
\hline Family physician & 25.7 & 26.1 & 25.5 & & 25.6 & 25.3 \\
\hline Cardiology & 41.4 & 40.5 & 14.9 & & 42.1 & 41.6 \\
\hline Internal medicine & 9.4 & 9.3 & 9.6 & & 9.1 & 10.4 \\
\hline Other & 6.6 & 6.8 & 6.5 & & 6.2 & 7.0 \\
\hline Missing & 16.9 & 17.4 & 16.5 & & 17.0 & 15.6 \\
\hline \multicolumn{7}{|l|}{ Hospital-level factors } \\
\hline $\begin{array}{l}\text { Annual no. of } \\
\text { catheterization } \\
\text { procedures, mean } \pm S D\end{array}$ & $4092.3 \pm 1696.4$ & $4031.3 \pm 1684.3$ & $4130.9 \pm 1702.9$ & $<0.001$ & $4120.9 \pm 1702.6$ & $4149.5 \pm 1703.6$ \\
\hline Hospital type & & & & $<0.001$ & & \\
\hline Catheterization only & 13.2 & 13.6 & 13.0 & & 13.4 & 12.2 \\
\hline $\begin{array}{l}\mathrm{PCl} \text { and } \\
\text { catheterization only }\end{array}$ & 6.1 & 7.2 & 5.4 & & 5.0 & 6.1 \\
\hline $\begin{array}{l}\mathrm{CABG}, \mathrm{PCl} \text { and } \\
\text { catheterization }\end{array}$ & 80.8 & 79.3 & 81.7 & & 81.7 & 81.8 \\
\hline \multicolumn{7}{|c|}{$\begin{array}{l}\mathrm{CABG}=\text { coronary artery bypass grafting, Cath = catheterization, } \mathrm{CCS}=\text { Canadian Cardiovascular Society, } \mathrm{COPD}=\text { chronic obstructive pulmonary disease, } \mathrm{ECG}= \\
\text { electrocardiogram, } \mathrm{LAD}=\text { left anterior descending, } \mathrm{LM}=\text { left main, } \mathrm{LV}=\text { left ventricular, } \mathrm{MI}=\text { myocardial infarction, } \mathrm{NA}=\text { not done, } \mathrm{PCl}=\text { percutaneous coronary } \\
\text { intervention, } \mathrm{PVD}=\text { peripheral vascular disease, } \mathrm{RCA}=\text { right coronary artery. } \\
\text { *Unless stated otherwise. } \\
\dagger \mathrm{LM} \text { if } \geq 50 \% \text { stenosis, proximal } L A D \text { if } \geq 70 \% \text { stenosis, mid } / \text { distal } L A D \text { if } \geq 70 \% \text { stenosis, circumflex if } \geq 70 \% \text { stenosis, } R C A \text { if } \geq 70 \% \text { stenosis. }\end{array}$} \\
\hline
\end{tabular}

method to determine the costs for emergency department visits and same-day surgeries, both using the National Ambulatory Care Reporting System database. We adjusted costs to 2013 Canadian dollars using the consumer price index.

\section{Statistical analyses}

We used hierarchical generalized linear models, with a logarithmic link and $\gamma$ distribution. The models incorporated hospital-specific random effects to account for within-hospital homogeneity in outcomes. The logarithmic link function is advantageous because it restricts predicted costs to positive values. In addition, final model coefficients are straightforward to interpret; specifically, the exponential of the coefficient provides a cost ratio, or the relative increase in the mean cost for a one unit change in the predictor variable. We used the $\gamma$ distribution because of the skewed distribution of health care costs. ${ }^{17}$ Our models included 2 main covariates of interest: the treatment allocation (medical v. percutaneous coronary intervention v. coronary artery bypass grafting) and the revascularization ratio (high v. medium v. low). In addition, the models included an interaction between treatment allocation and revascularization ratio. We adjusted the models for patient, physician and hospital factors, including patient demographics, cardiac morbidities, risk factors, diagnostic test results, coronary anatomy, 


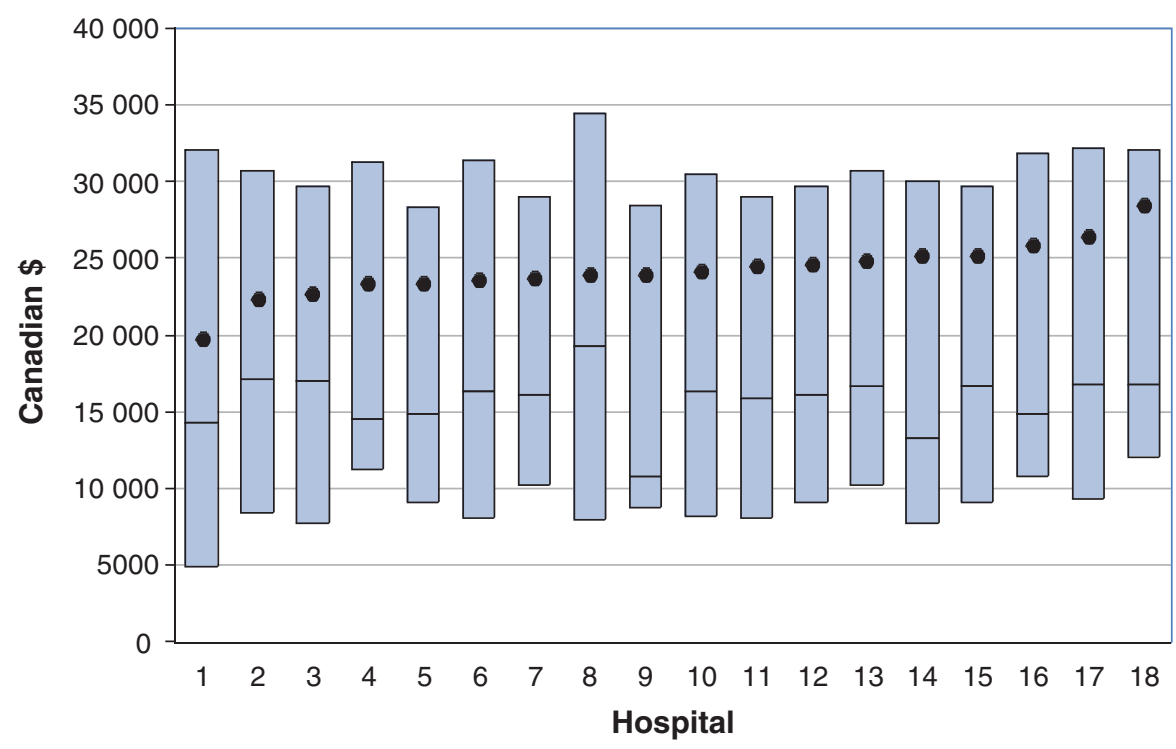

Figure 2: Variation in individual- and hospital-level costs. The median is plotted for each centre as a horizontal line, the boxes represent the interquartile range, and the circles represent the mean.

\begin{tabular}{|c|c|c|c|c|c|c|}
\hline \multirow[b]{2}{*}{ Category } & \multicolumn{3}{|c|}{ Cost, \$ } & \multirow{2}{*}{$\begin{array}{c}p \text { value } \\
\text { (medical therapy v. } \\
\text { revascularization) }\end{array}$} & \multicolumn{2}{|c|}{ Cost, \$ } \\
\hline & Total & Medical & Revascularization & & CABG & $\mathrm{PCl}$ \\
\hline $\begin{array}{l}\text { Acute care hospital } \\
\text { admission }\end{array}$ & 11373 & 7038 & 14109 & $<0.001$ & 25927 & 7755 \\
\hline Emergency department & 372 & 367 & 375 & 0.3 & 403 & 361 \\
\hline Surgery & 2691 & 2090 & 3071 & $<0.001$ & 1901 & 3699 \\
\hline Physician visits & 5449 & 4079 & 6313 & $<0.001$ & 10086 & 4284 \\
\hline Medication* & 1810 & 1857 & 1780 & 0.004 & 1476 & 1944 \\
\hline Laboratory & 194 & 195 & 193 & 0.3 & 217 & 180 \\
\hline Long-term care & 54 & 93 & 29 & $<0.001$ & 47 & 19 \\
\hline
\end{tabular}

referral physician specialization and hospital catheterization volume. We used SAS Version 9.3 for all analyses.

\section{Results}

\section{Patients}

Between Oct. 1, 2008, and Sept. 30, 2011, a total of 183630 angiography procedures were performed in Ontario. The final cohort included 39126 patients with stable coronary artery disease, of which 15138 received medical treatment and 23988 received revascularization (percutaneous coronary intervention: 15601 patients; coronary artery bypass grafting: 8387 patients; Figure 1). There were substantial differences in the baseline characteristics between medical therapy and revascularization groups (Table 1); patients who received medical treatment were older, more likely to be female, had greater comorbidity and had less severe symptoms of angina based on the classification of the Canadian Cardiovascular Society.

\section{Revascularization ratio}

The average revascularization ratio across the 18 Ontario hospitals was 1.58, ranging from 1.09 to 2.31 (Appendix 1 [Appendix Figure 1]).

\section{Variation in cumulative 1-year health care cost}

Individual patient 1 -year cost varied substantially (interquartile range \$8235-\$30 511). As seen in Figure 2, the overall cost was heavily skewed, with an overall median of $\$ 15707$ and overall mean of \$24 026. The mean costs for medical and revascularization treatments were \$18 069 (median \$7867) and 


\section{OPEN}

Research

\$27 786 (median \$21 428), respectively. The mean cost by hospital ranged from $\$ 19749$ to $\$ 28473$.

\section{Components of health care costs}

Most health care costs were due to acute care hospital admission, with a significantly higher cost for patients undergoing revascularization than for patients receiving medical therapy (Table 2). This was primarily driven by the cost of coronary artery bypass grafting.

\section{Predictors of health care costs}

The results of the fully adjusted model are found in Table 3. With respect to our 2 primary covariates of interest, there was no significant difference in cost associated across the tertiles of hospital revascularization ratio. In contrast, revascularization was a significant predictor of mean cost regardless of the modality (percutaneous coronary intervention: cost ratio 1.27, 95\% confidence interval [CI] 1.24-1.31; coronary artery bypass grafting: cost ratio $2.62,95 \%$ CI $2.53-2.71$ ).

In addition, sex was a statistically significant predictor of mean cost, with a cost ratio of 0.96 (95\% CI 0.95-0.98) for men, indicating that the mean per-patient cost was $4 \%$ less for men than for women (Table 3). Peripheral vascular disease (cost ratio $1.25,95 \%$ CI 1.21-1.28), hypertension (cost ratio $1.11,95 \%$ CI 1.08-1.13), and higher Charlson score (cost ratio 1.17, 95\% CI 1.16-1.17) were associated with higher mean cost. Previous cardiac surgery was associated with a $17 \%$ reduction in mean cost. Symptom severity, as measured by the Canadian Cardiovascular Society class, had an impact on mean cost, with more symptomatic patients, in general, having a lower mean cost. The specialty of the referral physician was a predictor of cost, with patients who were referred by either cardiologists or other physicians having a higher 1-year cumulative cost than those referred by family physicians. In general, busier hospitals (as measured by their annual cardiac catheterization volume) had lower cost, although the magnitude of this effect was small.

In our interaction model (Appendix 1 [Appendix Table 1]), we were able to compare the effect of each initial strategy on total costs, stratified by the type of hospital (low, medium or high revascularization ratio). We found that, on average, there was no difference in cumulative 1-year health care cost among patients who received medical treatment compared with those undergoing revascularization at the different strata defined by the hospital revascularization ratio. Similarly, patients who initially received percutaneous coronary intervention had similar costs at hospitals with high versus medium versus low revascularization ratios, as did patients who received coronary artery bypass grafting.

\section{Interpretation}

We found significant variation in 1-year cumulative health care costs among patients with stable coronary artery disease who received treatment at different hospitals. Greater patient comorbidity and treatment with revascularization were associated with higher cost. Importantly, the variation observed between hospitals based on revascularization ratio was not associated with a significant difference in cumulative
Table 3 (part 1 of 2): Predictors of 1-year cumulative health care costs

Covariate Cost ratio $(95 \% \mathrm{Cl})$

Treatment*

Medical management

Reference

$\mathrm{PCl}$

$1.27(1.24-1.31)$

CABG

$2.62(2.53-2.71)$

Patient-level factors

Demographic characteristics

\begin{tabular}{lc}
\hline Age, yr & $1.01(1.01-1.01)$ \\
\hline Sex, male & $0.96(0.95-0.98)$ \\
\hline Rural residence & $0.97(0.95-0.99)$
\end{tabular}

Income quintile

\begin{tabular}{|lc|}
\hline 5 (highest) & Reference \\
\hline 1 (lowest) & $1.10(1.07-1.12)$ \\
\hline 2 & $1.04(1.02-1.07)$ \\
\hline 3 & $1.02(1.00-1.05)$ \\
\hline 4 & $1.02(1.00-1.05)$ \\
\hline
\end{tabular}

Medical comorbidities

Peripheral vascular disease

$1.25(1.21-1.28)$

Previous MI

0.95 (0.93-0.97)

COPD

$1.10(1.07-1.13)$

Charlson score

$1.17(1.16-1.17)$

Cardiac risk factors

Diabetes

$1.00(0.99-1.02)$

Hypertension

$1.11(1.08-1.13)$

Hyperlipidemia

0.95 (0.93-0.97)

History of smoking

1.00 (0.99-1.02)

Cardiac status/testing

Native stenosis†

\begin{tabular}{|lc|}
\hline LM & $1.12(1.09-1.15)$ \\
\hline Proximal LAD & $1.10(1.08-1.11)$ \\
\hline Mid/distal LAD & $1.08(1.06-1.09)$ \\
\hline Circumflex & $1.11(1.09-1.12)$ \\
\hline RCA & $1.09(1.07-1.11)$ \\
\hline Previous CABG & $0.83(0.82-0.85)$ \\
\hline LV function, \% & Reference \\
\hline$\geq 50$ & $1.03(1.00-1.05)$ \\
\hline $35-49$ & $1.14(1.10-1.18)$ \\
\hline 34 & $0.95(0.93-0.97)$ \\
\hline NA &
\end{tabular}




\begin{tabular}{|c|c|}
\hline \multicolumn{2}{|c|}{$\begin{array}{l}\text { Table } 3 \text { (part } 2 \text { of 2): Predictors of 1-year cumulative health } \\
\text { care costs }\end{array}$} \\
\hline Covariate & Cost ratio $(95 \% \mathrm{Cl})$ \\
\hline \multicolumn{2}{|l|}{ Patient-level factors cont'd } \\
\hline \multicolumn{2}{|l|}{ Cardiac status/testing cont'd } \\
\hline \multicolumn{2}{|l|}{ Exercise ECG risk } \\
\hline Low risk & Reference \\
\hline High risk & $1.04(1.02-1.06)$ \\
\hline Uninterpretable & $1.09(1.05-1.14)$ \\
\hline NA & $1.24(1.21-1.27)$ \\
\hline \multicolumn{2}{|l|}{ Functional imaging risk } \\
\hline Low risk & Reference \\
\hline High risk & $1.01(0.99-1.03)$ \\
\hline Unknown/NA & $1.05(1.03-1.08)$ \\
\hline \multicolumn{2}{|l|}{ CCS class } \\
\hline 0 & Reference \\
\hline 1 & $0.90(0.87-0.92)$ \\
\hline 2 & $0.88(0.85-0.90)$ \\
\hline 3 & $0.90(0.88-0.92)$ \\
\hline 4 & $0.86(0.82-0.90)$ \\
\hline \multicolumn{2}{|l|}{ Physician factors } \\
\hline \multicolumn{2}{|l|}{ Referral physician } \\
\hline Family physician & Reference \\
\hline Cardiology & $1.02(1.01-1.05)$ \\
\hline Internal medicine & $1.00(0.98-1.03)$ \\
\hline Other & $1.22(1.19-1.27)$ \\
\hline Missing & $1.03(1.00-1.05)$ \\
\hline \multicolumn{2}{|l|}{ Hospital factors } \\
\hline \multicolumn{2}{|l|}{ Hospital revascularization ratio* } \\
\hline Low & Reference \\
\hline Medium & $1.03(0.93-1.15)$ \\
\hline High & $1.08(0.97-1.21)$ \\
\hline Annual catheterization volume & $0.99(0.99-0.99)$ \\
\hline \multicolumn{2}{|c|}{$\begin{array}{l}\text { Note: } C A B G=\text { coronary artery bypass grafting, } \mathrm{Cl}=\text { confidence interval, } C O P D= \\
\text { chronic obstructive pulmonary disease, } E C G=\text { electrocardiography, } L A D=\text { left } \\
\text { anterior descending, } L M=\text { left main, } L V=\text { left ventricular, } M I=\text { myocardial } \\
\text { infarction, } N A=\text { not done or missing, } P C l=\text { percutaneous coronary intervention, } \\
R C A=\text { right coronary artery. } \\
{ }^{*} T \text { The } 2 \text { main covariates of interest: treatment type and hospital revascularization } \\
\text { ratio. } \\
\dagger L M \text { if } \geq 50 \% \text { stenosis, proximal } L A D \text { if } \geq 70 \% \text { stenosis, mid/distal } L A D \text { if } \geq 70 \% \\
\text { stenosis, circumflex if } \geq 70 \% \text { stenosis, } R C A \text { if } \geq 70 \% \text { stenosis. }\end{array}$} \\
\hline
\end{tabular}

health care costs, regardless of the management strategy.

It has previously been reported that the direct cost of stable coronary artery disease accounted for $1.3 \%$ of UK national health expenditures. ${ }^{18}$ The vast majority of this cost was related to revascularization procedures (35\% of cost) and hospital bed occupancy (31\% of cost). Additionally, drug treatment accounted for $12 \%$ of total expenditures. Similar findings were found in several other UK and European studies. ${ }^{19,20}$

In our study, the major driver for 1-year cumulative costs was the receipt of revascularization. If revascularization offers no impact on mortality and if symptom relief is equivocal, as previous randomized controlled trials have shown, then our study suggests that the decision to pursue a revascularization strategy will translate into a substantial impact on health care resources. It follows that revascularization in patients with minimal symptoms in whom clinical benefit may be marginal may concurrently place an important financial burden on the health care system. This reinforces the importance of appropriate selection of patients who should receive percutaneous coronary intervention or coronary artery bypass grafting. Interestingly, the cumulative cost for each of the treatment strategies did not vary with hospital revascularization ratio - that is, the cost of a strategy was similar regardless of the tendencies of the site to favour revascularization or medical management. This implies a similar degree of efficiency for hospitals at different revascularization ratio strata, which is a reassuring finding. We also identified a number of patient-level factors that were associated with increased health care costs, including sex and previous coronary artery bypass grafting. We can only hypothesize as to the reasons for these findings. Regarding sex, men may have less health-seeking behaviour, which translated into a small difference in overall costs. For patients with previous coronary artery bypass grafting, we anticipate that these patients were more likely to be treated medically or by percutaneous coronary intervention rather than redoing surgery, resulting in lower overall costs.

\section{Limitations}

Our findings should be interpreted in the context of several limitations. First, this study did not take on a societal perspective, but rather that of the third-party payer for the province. Therefore, we excluded some potential costs, such as patient out-of-pocket and lost-productivity costs, ${ }^{19}$ and medication costs for patients younger than 65 years, as Ontario only provides comprehensive prescription drug coverage for patients 65 years of age or older. As such, total costs are skewed toward older patients. However, given that most patients in our cohort were over 65 years of age and, importantly, the bulk of costs were nonmedication related, this would not have made a qualitative difference in our conclusions. Second, there were substantial differences in patients who received medical treatment versus revascularization. Patients who received medical treatment were older, and were more likely to be female and have greater comorbidity. This may suggest that patients who receive medical treatment are more complex and more likely to be at higher risk of morbidity and mortality. Third, we were limited to evaluating covariates that were captured in the databases available. Other factors may have influenced patient costs. For example, aspects of physician and hospi- 
tal culture may have influenced patients' treatment strategy and, therefore, costs. Fourth, our cohort was accrued from 2008 to 2011, which was the most recent data available at the time of our study. We acknowledge the data are more than 5 years old; however, the interventions and practice patterns studied here are well-established, and we do not expect that more recent data would be significantly different because care for these patients has not changed. Fifth, based on our allocation of treatment strategy, there is a risk of survivorship bias, in that patients who died within 90 days were allocated to the medical-therapy group and thus have lower costs. This risk is mitigated by the fact that our cohort was a stable coronary artery disease population with very low mortality - indeed, our previous work showed a 90-day mortality of less than $1 \% .^{21}$ Finally, we limited our analyses to health care costs over a restricted 1-year time horizon in a cohort of patients after coronary angiography. As such, our results cannot be generalized to stable patients who have not undergone angiography. The use of a short time horizon may bias results against coronary artery bypass grafting, because it is associated with fewer revascularizations in the long term. However, these long-term costs would be discounted and therefore have a substantially smaller effect on overall costs.

\section{Conclusion}

Most costs associated with stable coronary artery bypass grafting were due to acute care hospital admission, with the primary driver being revascularization. Our study highlights the resource implications of an initial revascularization strategy.

\section{References}

1. Thériault L, Stonebridge C, Browarski S; Conference Board of Canada; Canadian Heart Health Strategy and Action Plan; Heart and Stroke Foundation of Canada; et al. The Canadian heart bealth strategy: risk factors and future cost implications. Ottawa: Conference Board of Canada; 2010.

2. Economic burden of illness in Canada, 2005-2008. Report no. 130148. Ottawa: Public Health Agency of Canada; 2014. Available: www.phac-aspc. gc.ca/publicat/ebic-femc/2005-2008/assets/pdf/ebic-femc-2005-2008-eng. pdf (accessed 2016 June 1).

3. Economic burden of illness in Canada, 1998. Ottawa: Health Canada; 2002. Available: http://publications.gc.ca/collections/Collection/H21-136-1998E. pdf (accessed 2016 June 1).

4. Simoons ML, Windecker S. Controversies in cardiovascular medicine: chronic stable coronary artery disease: drugs vs. revascularization. Eur Heart 7 2010;31:530-41.

5. Boden WE, O'Rourke RA, Teo KK, et al. Impact of optimal medical therapy with or without percutaneous coronary intervention on long-term cardiovascular end points in patients with stable coronary artery disease (from the COURAGE Trial). Am 7 Cardiol 2009;104:1-4.

6. BARI 2D Study Group; Frye RL, August P, Brooks MM, et al. A randomized trial of therapies for type 2 diabetes and coronary artery disease. $N$ Engl $7 \mathrm{Med}$ 2009;360: 2503-15.

7. Bennell MC, Qiu F, Kingsbury KJ, et al. Determinants of variations in initial treatment strategies for stable ischemic heart disease. CMA7 2015;187:E317-25.

8. Anderson JL, Heidenreich PA, Barnett PG, et al. ACC/AHA statement on cost/ value methodology in clinical practice guidelines and performance measures: a report of the American College of Cardiology/American Heart Association Task Force on Performance Measures and Task Force on Practice Guidelines. $7 \mathrm{Am}$ Coll Cardiol 2014;63:2304-22.

9. Cardiac Care Network of Ontario [homepage]. Available: www.ccn.on.ca/ ccn_public/FormsHome/HomePage.aspx (accessed 2016 June 1).

10. Annual report 2001-2012. Toronto: Cardiac Care Network of Ontario; 2012. Available: www.ccn.on.ca/ccn_public/uploadfiles/files/CCN_Annual _Report_2012.pdf (accessed 2016 June 1).

11. Ko DT, Guo H, Wijeysundera HC, et al.; Cardiac Care Network (CCN) of Ontario Variations in Revascularization Practice in Ontario (VRPO) Working Group. Assessing the association of appropriateness of coronary revascularization and clinical outcomes for patients with stable coronary artery disease. 7 Am Coll Cardiol 2012;60:1876-84.
12. Schwalm JD, Wijeysundera HC, Tu JV, et al. Influence of coronary anatomy and SYNTAX Score on the variations in revascularization strategies for patients with multivessel disease. Can 7 Cardiol 2014;30:1155-61.

13. Tu JV, Ko DT, Guo H, et al.; Cardiac Care Network of Ontario's Variations in Revascularization Practice in Ontario Working Group. Determinants of variations in coronary revascularization practices. CMA7 2012;184:179-86

14. Gurevich Y, McFarlane A, Morris K, et al. Estimating the number of coronary artery bypass graft and percutaneous coronary intervention procedures in Canada: A comparison of cardiac registry and Canadian Institute for Health Information data sources. Can 7 Cardiol 2010;26:e249-53.

15. Wijeysundera HC, Wang X, Tomlinson G, et al. Techniques for estimating health care costs with censored data: an overview for the health services researcher. Clinicoecon Outcomes Res 2012;4:145-55.

16. Jacobs P, Yim R. Using Canadian administrative databases to derive economic data for bealth technology assessments. Ottawa: Canadian Agency for Drugs and Technologies in Health; 2009. Available: www.cadth.ca/media/pdf/H0483 _Canadian_Admin_Databases_mg_e.pdf (accessed 2016 June 1).

17. Austin PC, Ghali $\bar{W} A, T u$ JV. A comparison of several regression models for analysing costs of CABG surgery. Stat Med 2003;22:2799-815.

18. Stewart S, Murphy NF, Walker A, et al. The current cost of angina pectoris to the National Health Service in the UK. Heart 2003;89:848-53.

19. Liu JL, Maniadakis N, Gray A, et al. The economic burden of coronary heart disease in the UK. Heart 2002;88:597-603.

20. Leal J, Luengo-Fernández R, Gray A, et al. Economic burden of cardiovascular diseases in the enlarged European Union. Eur Heart 7 2006;27:1610-9.

21. Wijeysundera HC, Bennell MC, Qiu F, et al. Comparative-effectiveness of revascularization versus routine medical therapy for stable ischemic heart disease: a population-based study. 7 Gen Intern Med 2014;29:1031-9.

Affiliations: Division of Cardiology (Kang, Bennell, Ko, Wijeysundera), Sunnybrook Health Sciences Centre, Schulich Heart Centre, University of Toronto; Institute for Clinical Evaluative Sciences (Qiu, Austin, Ko, Wijeysundera), Toronto, Ont.; Libin Cardiovascular Institute of Alberta (Knudtson), University of Calgary, Calgary, Alta.; Institute of Health Policy, Management and Evaluation (Austin, Ko, Wijeysundera), University of Toronto, Toronto, Ont.

Contributors: Jaskaran Kang and Maria Bennell contributed to the design of the study, and analysis and interpretation of data. Feng Qiu, Merril Knudtson, Peter Austin and Dennis Ko contributed to the analysis and interpretation of data. Harindra Wijeysundera contributed to the conception and design of the study, and analysis and interpretation of data. Jaskaran Kang drafted the article, which all of the other authors revised for important intellectual content. All of the authors gave final approval of the version to be published and agree to act as guarantors of the work.

Funding: The clinical registry data used in this publication are from the Cardiac Care Network of Ontario and its member hospitals. The Cardiac Care Network of Ontario is dedicated to improving the quality, efficiency, access and equity of adult cardiovascular services in Ontario, Canada. The Cardiac Care Network of Ontario serves as an advisory body to the Ontario Ministry of Health and Long-Term Care (MOHLTC). This study was funded in part by operating funds from the Canadian Institutes of Health Research (CIHR), Schulich Heart Centre and the Sunnybrook Research Institute. This study was supported by the Institute for Clinical Evaluative Sciences (ICES), which is funded by an annual grant from the Ontario MOHLTC. The opinions, results and conclusions reported in this paper are those of the authors and are independent from the funding sources. No endorsement by ICES or the Ontario MOHLTC is intended or should be inferred. Parts of this material are based on data and information compiled and provided by the CIHR. However, the analyses, conclusions, opinions and statements expressed herein are those of the author and not necessarily those of the CIHR. Harindra Wijeysundera is supported by a Distinguished Clinical Scientist Award from the Heart and Stroke Foundation of Canada. Dennis Ko is supported by a Mid-Career Award from the Heart and Stroke Foundation, Ontario Provincial Office. Peter Austin is supported in part by a Career Investigator Award from the Heart and Stroke Foundation.

Supplemental information: For reviewer comments and the original submission of this manuscript, please see www.cmajopen.ca/content/4/3/ E409/suppl/DC1.

ICES Disclaimer: This study was supported by the Institute for Clinical Evaluative Sciences (ICES) which is funded by an annual grant from the Ontario Ministry of Health and Long-Term Care (MOHLTC). The opinions, results and conclusions reported in this article are those of the authors and are independent from the funding sources. No endorsement by ICES or the Ontario MOHLTC is intended or should be inferred. 